\title{
Bilateral Tuberculous Pleurisy with Subsequent Upper Lobe Predominant Pulmonary Fibrosis Mimicking Pleuroparenchymal Fibroelastosis
}

\author{
Takako Kawaguchi ${ }^{1}$, Keishi Oda ${ }^{1}$, Takashi Kido ${ }^{1}$, Toshinori Kawanami ${ }^{1}$, \\ Yoshinori Kawabata ${ }^{2}$ and Kazuhiro Yatera ${ }^{1}$
}

\begin{abstract}
:
Although the majority of patients with Mycobacterium tuberculosis have pulmonary involvement, some cases have pleural involvement as extra-pulmonary sites of infection. We herein report a case of upper lobepredominant pulmonary fibrosis that developed in a 47-year-old male with a history of bilateral tuberculous pleurisy. Based on his chest radiological findings, pleuroparenchymal fibroelastosis (PPFE) was most strongly suspected, and a surgical lung biopsy (SLB) was performed to obtain a pathological diagnosis. The SLB specimens showed interstitial pneumonia with pleural involvement without any characteristic findings of PPFE. Careful discretion in obtaining a precise diagnosis of this condition should be practiced in such cases.
\end{abstract}

Key words: tuberculous pleurisy, respiratory failure, pleuroparenchymal fibroelastosis, pleural effusion

(Intern Med 57: 85-89, 2018)

(DOI: 10.2169/internalmedicine.9187-17)

\section{Introduction}

Mycobacterium tuberculosis is one of the most common causes of death worldwide, and the number of registered cases of primary tuberculosis still exceeds 18,000 a year in Japan (1). Approximately 5-20\% of those patients also develop extrapulmonary tuberculosis, and tuberculous pleurisy is the second-most common form of extra-pulmonary tuberculosis (2).

Tuberculous pleurisy is characterized by an intense chronic accumulation of pleural effusion and lymphocytepredominant inflammatory cells in the pleural space and it can cause clinical symptoms and pleural fibrosis with resultant residual pleural thickening. However, a previous study reported that these complicating disorders tend to decrease with time and have negligible functional consequences (3), although some cases occasionally show moderate to severe pulmonary function impairment.

We herein report a case of progressive respiratory failure after treatment for bilateral tuberculous pleurisy in which the clinical behavior and chest radiological findings very closely mimicked pleuroparenchymal fibroelastosis (PPFE), with a review of the relevant literature.

\section{Case Report}

A 47-year-old Japanese male who was non-smoker without any history of occupational dust exposure was referred to our hospital with complaints of persistent dry cough for a month associated with left chest pain and a low-grade fever. His initial chest X-ray demonstrated bilateral pleural effusion with left-side predominance (Fig. 1A), and he had undergone left thoracentesis. An analysis of the obtained left pleural fluid revealed a white blood cell count of 1,238 cells/ $\mathrm{LL}$ (predominantly lymphocytes, $75 \%$ ) with an adenosine deaminase level of $166.2 \mathrm{IU} / \mathrm{L}$. Staining for acid-fast bacilli in the pleural effusion was negative. Cultures of the pleural effusion were also negative for mycobacterial infections. A plasma interferon-gamma release assay revealed a positive result for Mycobacterium tuberculosis. Three sputum smears were negative for acid-fast bacilli. There are no

${ }^{1}$ Department of Respiratory Medicine, University of Occupational and Environmental Health, Japan, Japan and ${ }^{2}$ Division of Diagnostic Pathology, Saitama Prefectural Cardiovascular and Respiratory Center, Japan

Received: March 15, 2017; Accepted: May 10, 2017; Advance Publication by J-STAGE: October 16, 2017

Correspondence to Dr. Keishi Oda, oda-keishi@med.uoeh-u.ac.jp 

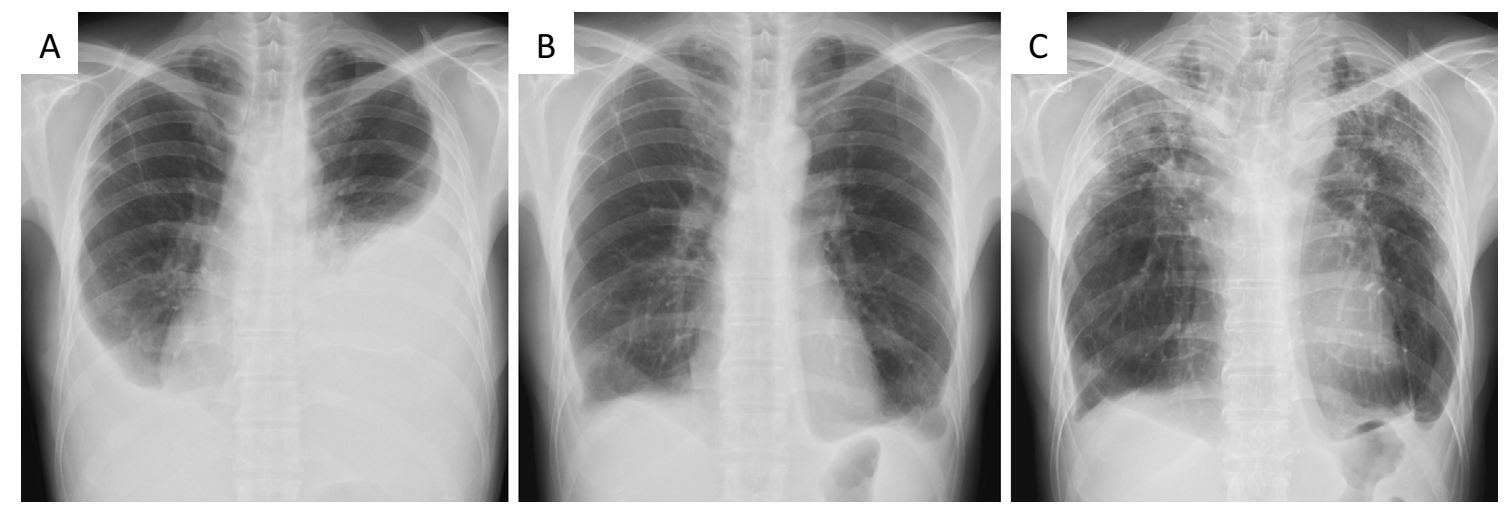

Figure 1. Chest X-ray findings at the first visit to our hospital showed left-side-predominant bilateral pleural effusions (A). After the treatment for tuberculous pleurisy, the bilateral pleural effusions completely disappeared (B). Multifocal consolidations and reticular-nodular infiltrations predominantly seen in the upper lobes were observed in the peripheral portions of both lungs three years after antituberculous treatment $(\mathrm{C})$.

specific CT findings for pulmonary tuberculosis. He then underwent a left pleural biopsy to establish a definitive diagnosis, and the results of acid-fast bacilli culture and polymerase chain reaction for $M$. tuberculosis of obtained pleural tissue were positive with histologic findings demonstrating the presence of many necrotizing and non-necrotizing granulomas (Fig. 2A and B). He was diagnosed with tuberculous pleurisy and started standard treatment with antituberculous agents (ethambutol, isoniazid, rifampicin and pyrazinamide). After 10 months of this treatment, a marked decrease in the bilateral pleural effusion was noted on chest X-rays (Fig. 1B), and all of his symptoms had completely improved.

Three years later, progressive and significant clinical and functional impairment with the development of exertional hypoxaemia was observed, and he was readmitted to our hospital due to worsening dyspnea on exertion. Chest X-rays (Fig. 1C) and high-resolution computed tomography (HRCT) (Fig. 3) showed bilateral apical pleural thickening associated with bronchiectasis, parenchymal bands, distortion and shrinkage in both upper lobes, as well as a flattened thoracic cage. The results of peripheral blood examinations, cultures of sputum and gastric fluid did not suggest a reactivation of tuberculous pleurisy. The serum levels of Krebs von den Lungen (KL)-6 and surfactant protein (SP)-D were $2,328 \mathrm{U} / \mathrm{mL}$ and $305 \mathrm{ng} / \mathrm{mL}$, respectively. Serum rheumatoid factor was negative, and the levels of antinuclear antibodies and immunoglobulins were normal. Pulmonary function tests were performed and showed a decrease within the previous 3 years of the forced expiratory volume in a second $\left(\mathrm{FEV}_{1}\right)$ from 2,960 to $730 \mathrm{~mL}$ (82 to $20 \%$ of predicted), forced vital capacity (FVC) from 3,240 to $770 \mathrm{~mL}$ (77 to $18 \%$ of predicted), $\mathrm{FEV}_{1} / \mathrm{FVC}$ from $91 \%$ to $95 \%$ and residual volume/total lung capacity $73 \%$ (223\% of predicted). An arterial blood analysis under resting conditions in the supine position on room air revealed partial pressure of arterial oxygen $\left(\mathrm{PaO}_{2}\right) 73$ Torr, partial pressure of carbon dioxide in arterial blood $\left(\mathrm{PaCO}_{2}\right)$ 57.2 Torr, base excess,
$\mathrm{HCO}_{3}-31.4 \mathrm{mEq} / \mathrm{L}$. Although a six-minute walk test was performed, he desaturated to $80 \%$ on room air after just 100 meters accompanied by severe dyspnea. Based on the findings of chest CT and negative findings of reactivation of tuberculous pleurisy, PPFE was suspected, and a videoassisted thoracoscopic surgical lung biopsy (SLB) was performed from his left upper lobe to make a definitive diagnosis. The degree of pleural adhesion was significant and desquamation of the pleural fibrosis was therefore considered to be surgically required. Cultures of the obtained lung tissues (left upper lobe) were negative for fungal, bacterial and mycobacterial infections. The pathological findings of the surgically-obtained lung tissues from the newly recognized opacity in the upper lobe were consistent with obstructive typed luminal organization without any collapse of the lung or elastosis located slightly apart from pleura, while the gradual transition to a normal lung was also observed (Fig. 2C and D). This lesion was diagnosed as unclassifiable interstitial pneumonia with massive luminal organization. Since discharge from our hospital, his symptoms and chest radiological findings have remained unchanged with no treatment, and an application for lung transplantation has been made and is currently under periodic review.

\section{Discussion}

The clinical behavior and chest radiological findings of the present case indicated a progression of restrictive lung disorder with upper lobe predominant pulmonary fibrosis that very strongly mimicked PPFE. However, the patient's history of treatment for bilateral tuberculous pleurisy and the pathological findings of lung specimens obtained from the left upper lobe including pleura were inconsistent with PPFE, and we were able to exclude other idiopathic interstitial pneumonias (IIPs), diffuse lung disease such as chronic hypersensitivity pneumonia, infectious disease and malignant disease. To our knowledge, this is the first report discussing the association of progressive interstitial pneumonia with bi- 

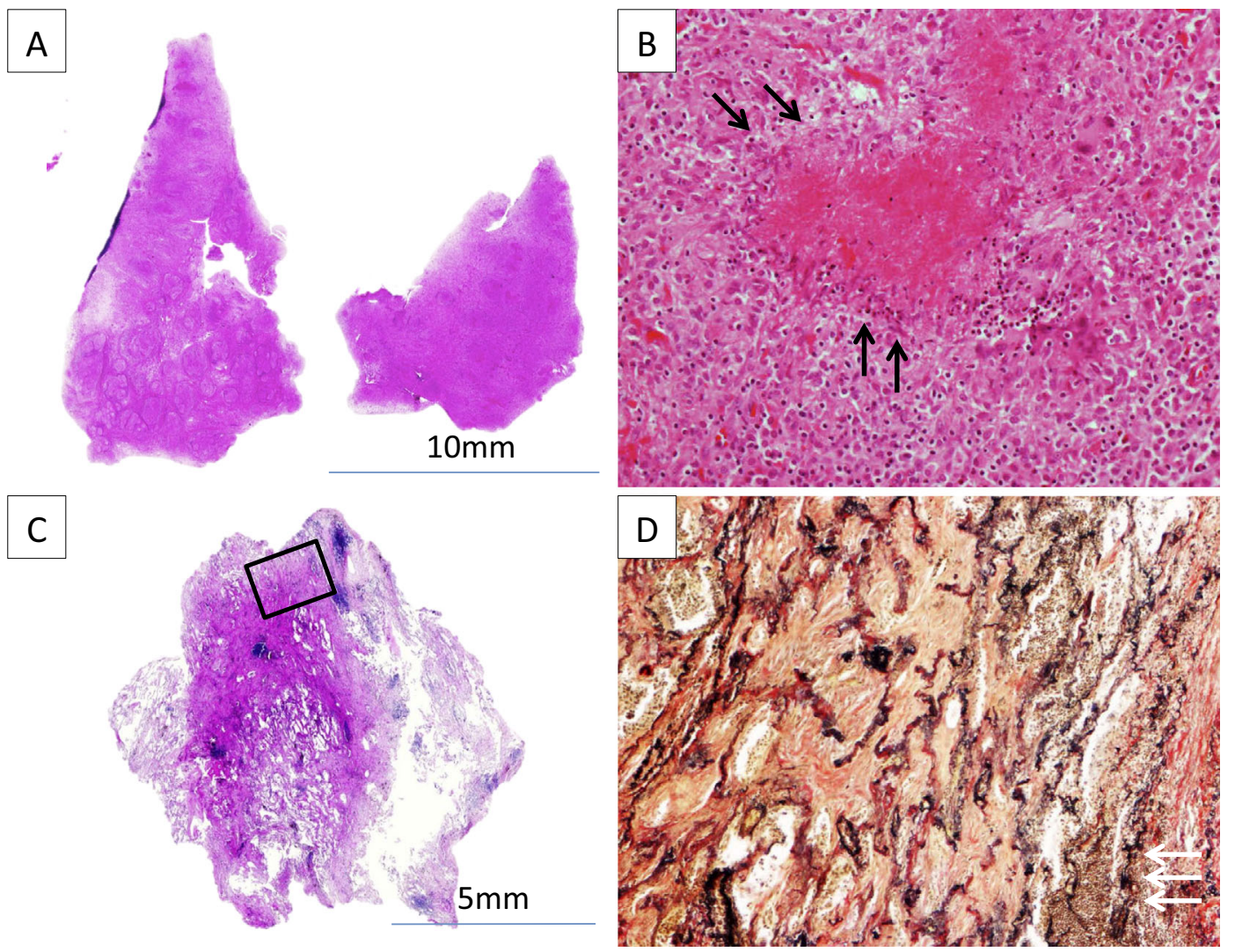

Figure 2. Pleura showed marked fibrosis containing many necrotizing and non-necrotizing granulomas. Bar $10 \mathrm{~mm}$. Hematoxylin and Eosin (H\&E) staining, Panoramic view (A). Central coagulation necrosis (Black arrows) surrounded by epithelioid cell layer. H\&E staining, $\times 200$ (B). A nodular lesion with gradual transition toward normal lung. Bar 5 mm. H\&E staining, Panoramic view (C). Massive obstructive typed luminal organization without collapse or elastosis. White arrows indicated pleural elastic layer. Elastic van Gieson staining, $\times 80$ (D).
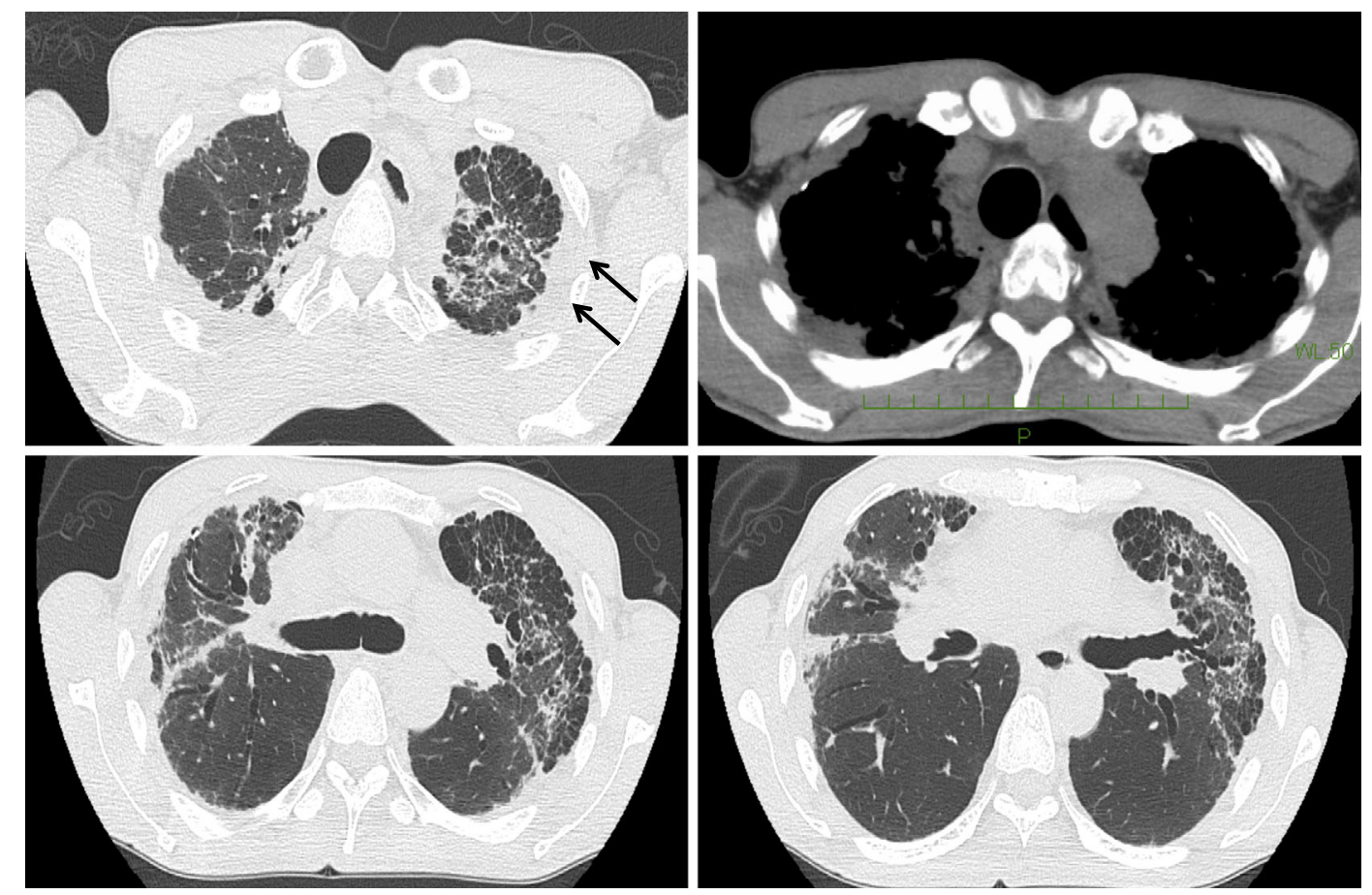

Figure 3. High-resolution computed tomography revealed features of pleuroparenchymal fibroelastosis at the lung apices with pleural thickening and traction bronchiectasis. The arrows indicate the surgical biopsy site. 
lateral tuberculous pleurisy that mimics PPFE with pathological findings of surgically-obtained lung and pleura samples.

Tuberculous pleurisy usually presents as an acute illness and it often causes pleural effusion. Tuberculous pleurisy is recommended to be treated with a standard anti-tuberculous therapy using ethambutol, isoniazid, rifampicin and pyrazinamide, and this treatment can also relieve the patients' symptoms and development of fibrothorax (2). The pleural effusion due to tuberculous pleurisy usually resolves with proper treatment, resulting in normal chest radiographic findings or only with pleural thickening of several millimeters $(4,5)$. A previous study reported that the administration of fibrinolytic agents may decrease the degree of residual pleural thickening in patients with loculated tuberculous pleural effusion (6). The present case developed progressive respiratory failure with a restrictive pulmonary function impairment without treatment other than anti-tuberculous therapy, and the clinical course and chest radiological findings were very similar to PPFE.

PPFE is a type of interstitial pneumonia first described in 2004 histologically characterized by upper lobe-predominant volume loss, pleural thickening and predominant subpleural fibroelastosis (7). Idiopathic PPFE was defined as a rare form of IIP in the latest international multidisciplinary classification of IIPs (8). In addition, several medications, such as chemotherapy agents, and bone marrow transplantation can be etiological of PPFE (9). PPFE has also been associated with a variety of clinicopathological conditions, such as autoimmune disease $(7,10,11)$, cancer $(12)$, prior exposure to metal dust (13-15), and infection, including pulmonary tuberculous $(12,16,17)$. The radiological findings of PPFE in HRCT are the presence of upper lobe pleural thickening and subpleural fibrosis associated with less marked or absent lower lobe involvement. A histological diagnosis of PPFE is made when well defined intraalveolar fibrosis with extensive alveolar septal elastosis and lung collapse is present with various degrees of pleural fibrosis (10). Nakatani et al. reported that coexistent interstitial lung disease apart from fibroelastosis was present in about $75 \%$ of PPFE cases (11).

The characteristic pathological features of the present case were luminal organization without elastosis or lung collapse slightly apart from pleura at the left upper lobe. In addition, macroscopically severe pleural inflammatory and adhesive changes were observed which were considered to potentially lead to a progression of restrictive pulmonary impairment. Although these findings are unusualy for PPFE, Hitora et al. reported that some inflammatory or acute lung injury might be a first step in the development and occurrence of PPFE (18). In addition, the findings of these biopsy specimens were thought to have the potential of developing into PPFE in the future.

The relationship between the treatment history of bilateral tuberculous pleuritis and upper lobe-predominant interstitial pneumonia is unknown, [only a few reports of drug-induced pneumonia caused by antituberculous agents $(19,20)]$ and the suitability of a diagnosis of unclassifiable interstitial pneumonia for this patient and categorizing the old pleural changes due to bilateral tuberculous pleuritis may be controversial. In addition, $M$. tuberculosis as the cause of bilateral pleuritis may one reason this disease mimics PPFE, as the progressive manifestation of unilateral tuberculous pleuritis may be a major marker differentiating tuberculous pleuritis and PPFE. Bilateral upper lobe pleural thickening and subpleural fibrosis associated with less marked or absent lower lobe involvement in cases with Mycobacterium tuberculosis infections have been reported (21), and cases similar to the present patient may exist. The careful clinical identification of a possible relationship between PPFE-like changes after tuberculous pleurisy is necessary by physicians, in order to prevent the spread of the disease entity of PPFE in the future. The pathogenesis and effective treatment of the present case with progressive respiratory failure after bilateral tuberculous pleurisy remain unclear, and lung transplantation may be one potential treatment strategy in advanced patients. Steroid therapy was not used for the present case, because there was a potential risk of recurrent tuberculosis and pneumothorax and no established beneficial effect of steroid therapy has so far been reported in cases of chronic interstitial pneumonia, including the present case. The present case did not show the pathological characteristics of PPFE, despite its clinical behavior mimicking that of PPFE. In addition, we were unable to obtain sufficient lung and pleural tissue specimens to perforom a pathological evaluation, as there was also adhesion in the pleural cavity, which may be one limitation associated with the present case. Further information with the accumulation of similar cases and pathological findings will be necessary to determine the mechanism underlying how bilateral tuberculous pleurisy-induced lung and pleural changes inducing interstitial pneumonia mimicked the clinical course and radiological findings of PPFE in this rare case.

The authors state that they have no Conflict of Interest (COI).

\section{References}

1. World Health Organization. Global Tuberculosis Report 2016. In: WHO/HTM/TB/2016.13. World Health Organization, Geneva, 2016.

2. Light RW. Update on tuberculous pleural effusion. Respirology 15: 451-458, 2010

3. Chung CL, Chen CH, Yeh CY, Sheu JR, Chang SC. Early effective drainage in the treatment of loculated tuberculous pleurisy. Eur Respir J 31: 1261-1267, 2008.

4. de Pablo A, Villena V, Echave-Sustaeta J, Encuentra AL. Are pleural fluid parameters related to the development of residual pleural thickening in tuberculosis? Chest 112: 1293-1297, 1997.

5. Barbas CS, Cukier A, de Varvalho CR, Barbas Filho JV, Light RW. The relationship between pleural fluid findings and the development of pleural thickening in patients with pleural tuberculosis. Chest 100: 1264-1267, 1991.

6. Cases Viedma E, Lorenzo Dus MJ, Gonzalez-Molina A, Sanchis Aldas JL. A study of loculated tuberculous pleural effu- 
sions treated with intrapleural urokinase. Respir Med 100: 20372042, 2006.

7. Frankel SK, Cool CD, Lynch DA, Brown KK. Idiopathic pleuroparenchymal fibroelastosis: description of a novel clinicopathologic entity. Chest 126: 2007-2013, 2004.

8. Travis WD, Costabel U, Hansell DM, et al. An official American Thoracic Society/European Respiratory Society statement: Update of the international multidisciplinary classification of the idiopathic interstitial pneumonias. Am J Respir Crit Care Med 188: 733-748, 2013.

9. Watanabe K. Pleuroparenchymal fibroelastosis: its clinical characteristics. Curr Respir Med Rev 9: 229-237, 2013.

10. Reddy TL, Tominaga M, Hansell DM, et al. Pleuroparenchymal fibroelastosis: a spectrum of histopathological and imaging phenotypes. Eur Respir J 40: 377-385, 2012.

11. Nakatani $T$, Arai $T$, Kitaichi $M$, et al. Pleuroparenchymal fibroelastosis from a consecutive database: a rare disease entity? Eur Respir J 45: 1183-1186, 2015.

12. Baroke E, Heussel CP, Warth A, et al. Pleuroparenchymal fibroelastosis in association with carcinomas. Respirology 21: 191-194, 2016.

13. Piciucchi S, Tomassetti S, Casoni G, et al. High resolution CT and histological findings in idiopathic pleuroparenchymal fibroelastosis: features and differential diagnosis. Respir Res 12: 111, 2011.

14. Becker CD, Gil J, Padilla ML. Idiopathic pleuroparenchymal fibroelastosis: an unrecognized or misdiagnosed entity? Mod Pathol 21: 784-787, 2008.

15. Inuzuka K, Yasui M, Waseda Y, Takato H, Ichikawa Y, Fujimura
M. [A case of repeated bilateral pneumothorax associated with upper lobe predominant fibrosis in an aluminum processing worker]. Nihon Kokyuki Gakkai Zasshi 48: 492-496, 2010 (in Japanese, Abstract in English).

16. Hurtado EJ, Gonzalez ML, Soto Mdel M, Rueda FJ, Nadal FJ, Cantero AR. Idiopathic pleuroparenchymal fibroelastosis, a new idiopathic interstitial pneumonia: A case report. Chron Respir Dis 13: 312-316, 2016.

17. Machuca JS, Niazi M, Diaz-Fuentes G. Pleuroparenchymal fibroelastosis presenting as a hypermetabolic lung nodule. J Bronchology Interv Pulmonol 18: 65-68, 2011.

18. Hirota T, Yoshida Y, Kitasato Y, et al. Histological evolution of pleuroparenchymal fibroelastosis. Histopathology 66: 545-554, 2015.

19. Kunichika N, Miyahara N, Kotani K, Takeyama H, Harada M, Tanimoto M. Pneumonitis induced by rifampicin. Thorax 57: 1000-1001, 2002.

20. Salomaa ER, Ruokonen EL, Tevola K, Tala E. Pulmonary infiltrates and fever induced by isoniazid. Postgrad Med J 66: 647649, 1990.

21. Kawabata Y, Iwai K. [Tuberculosis sequelae: pathological findings]. Kekkaku 65: 839-845, 1990 (in Japanese, Abstract in English).

The Internal Medicine is an Open Access article distributed under the Creative Commons Attribution-NonCommercial-NoDerivatives 4.0 International License. To view the details of this license, please visit (https://creativecommons.org/licenses/ by-nc-nd/4.0/).

(C) 2018 The Japanese Society of Internal Medicine

Intern Med 57: 85-89, 2018 